\title{
Ground states solutions for modified fourth-order elliptic systems with steep well potential
}

\section{Liuyang Shao, Haibo Chen*}

School of Mathematics and Statistics, Central South University, Changsha, 410083 Hunan, P. R. China.

Communicated by D. Baleanu

\section{Abstract}

In this paper, we study the following modified quasilinear fourth-order elliptic systems

$$
\begin{cases}\triangle^{2} u-\triangle u+(\lambda \alpha(x)+1) u-\frac{1}{2} \triangle\left(u^{2}\right) u=\frac{p}{p+q}|u|^{p-2}|v|^{q} u, & \text { in } \mathbb{R}^{N}, \\ \triangle^{2} v-\triangle v+(\lambda \beta(x)+1) v-\frac{1}{2} \triangle\left(v^{2}\right) v=\frac{q}{p+q}|u|^{p}|v|^{q-2} v, & \text { in } \mathbb{R}^{N},\end{cases}
$$

where $\triangle^{2}=\triangle(\triangle)$ is the biharmonic operator, $\lambda>0$, and $2<p, 2<q, 4<p+q<22^{* *}, 2^{* *}=\frac{2 N}{N-4}(N \leqslant 5)\left(\right.$ if $\left.N \leqslant 4,2^{* *}=\infty\right)$ is the critical Sobolev exponent for the embedding $W^{2,2}\left(\mathbb{R}^{N}\right) \hookrightarrow \mathrm{L}^{2^{* *}}\left(\mathbb{R}^{N}\right)$. Under some appropriate assumptions on $\alpha(x)$ and $\beta(x)$, we obtain that the above problem has nontrivial ground state solutions via the variational methods. We also explore the phenomenon of concentration of solutions.

Keywords: Fourth-order elliptic, variational methods, ground state solutions, concentration.

2010 MSC: 35B09, 35J20.

\section{Introduction and main results}

In this paper, we consider the following modified quasilinear fourth-order elliptic systems

$$
\begin{cases}\triangle^{2} \mathfrak{u}-\triangle \mathfrak{u}+(\lambda \alpha(x)+1) \mathfrak{u}-\frac{1}{2} \triangle\left(\mathrm{u}^{2}\right) \mathfrak{u}=\frac{\mathrm{p}}{\mathrm{p}+\mathrm{q}}|\mathfrak{u}|^{p-2}|v|^{\mathrm{q}} \mathrm{u}, & \text { in } \mathbb{R}^{\mathrm{N}}, \\ \triangle^{2} v-\triangle v+(\lambda \beta(x)+1) v-\frac{1}{2} \triangle\left(v^{2}\right) v=\frac{\mathrm{q}}{\mathrm{p}+\mathrm{q}}|\mathfrak{u}|^{\mathrm{p}}|v|^{\mathrm{q}-2} v, & \text { in } \mathbb{R}^{N},\end{cases}
$$

where $\triangle^{2}=\triangle(\triangle)$ is the biharmonic operator. $\lambda>0$, and $2<p, 2<q, 4<p+q<22^{* *}, 2^{* *}=\frac{2 N}{N-4}(N \leqslant$ 5) (if $N \leqslant 4,2^{* *}=\infty$ ) is the critical Sobolev exponent for the embedding $W^{2,2}\left(\mathbb{R}^{N}\right) \hookrightarrow L^{2^{* *}}\left(\mathbb{R}^{N}\right)$. In the

\footnotetext{
${ }^{*}$ Corresponding author

Email addresses: 280970756@qq. com (Liuyang Shao), math_chb@163.com (Haibo Chen)
}

doi: $10.22436 /$ jnsa.011.03.01

Received: 2017-11-29 Revised: 2017-12-31 Accepted: 2018-01-07 
last several decades, many researchers considered the problem

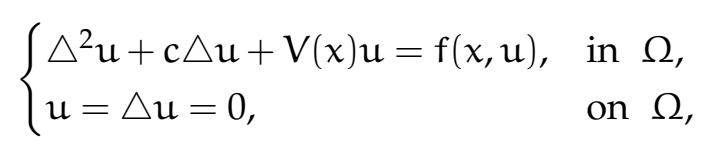

where $\Omega$ is a smooth bounded domain of $\mathbb{R}^{\mathrm{N}}$. Such equation arises in applications from mathematical physics. For instance, in [12], Lazer and McKenna studied the problem of nonlinear oscillation in a suspension bridge. They presented a mathematical model for the bridge that takes account of the fact that the coupling provided by the stays connecting the suspension cable to the deck of the road bed is fundamentally nonlinear. For more details, we refer the readers to $[6,13,17]$ and the references therein.

In recently years, with the aid of variational methods, the existence, nonexistence, and multiplicity results of various solutions for (1.1) have been extensively investigated (see [1-5, 7-11, 14-16, 18-23, 2632]). For example, in [19], Pimenta and Soares considered the superlinear fourth-order elliptic equation (1.1) and by concentrating at a point in the limit by using a weak version of the Ambrosetti-Rabinowitz condition proved th existence of a family of solutions. Furthermore, in [33], Zhang et al. proved the existence of nontrivial solutions and investigated the concentration phenomenon of solutions on the set $\mathrm{V}^{-1}(0)$. But to the best of our knowledge, little researches have been done for (1.1) with the quasilinear team $\int_{\mathbb{R}^{N}} u \triangle\left(u^{2}\right) d x$, because the appearance of the quasilinear team $\int_{\mathbb{R}^{N}} u \triangle\left(u^{2}\right) d x$, makes the studies more difficult and interesting.

Motivated by the above facts, the goal of this paper is to consider the ground state solutions for problem (1.1) with steep well potential. Under some natural assumptions for $\alpha(x)$ and $\beta(x)$, we obtain that the problem (1.1) has a nontrivial ground state solutions via the variation methods. Furthermore, we also explore the phenomenon of concentration of solutions.

Before stating our main results, we give the following assumption on $\alpha(x)$ and $\beta(x)$.

$\left(F_{1}\right) \alpha(x), \beta(x) \in C\left(\mathbb{R}^{N}, \mathbb{R}\right)$ satisfying $\alpha(x) \geqslant 0, \beta(x) \geqslant 0$, and $\Lambda=\operatorname{int}\left\{x \in R^{N}: \alpha(x)=0, \beta(x)=0\right\}$, is nonempty and has smooth boundary with $\bar{\Lambda}=\left\{x \in \mathbb{R}^{N}: \alpha(x)=0, \beta(x)=0\right\}$;

$\left(F_{2}\right)$ there exist $M_{1}, M_{2}>0$ such that

$$
\mathfrak{m}\left(\left\{x \in \mathbb{R}^{\mathrm{N}} \mid \alpha(x) \leqslant M_{1}\right\}\right)<\infty, \quad \text { and } \mathrm{m}\left(\left\{x \in \mathbb{R}^{\mathrm{N}} \mid \beta(x) \leqslant M_{2}\right\}\right)<\infty,
$$

where $m$ denotes the Lebesgue measure in $\mathbb{R}^{N}$.

For simplicity, in the rest of the paper, we denote $V_{1}(x)=\lambda \alpha(x)+1$, and $V_{2}(x)=\lambda \beta(x)+1$. We also make the following assumption

$\left(F_{0}\right) V_{i} \in C\left(\mathbb{R}^{N}, \mathbb{R}\right)$, and $\inf V_{i}(x) \geqslant 1>0$, moreover, there is a constant $d_{0}>0$ such that

$$
\lim _{|y| \rightarrow \infty} \operatorname{meas}\left\{x \in \mathbb{R}^{N}:|x-y| \leqslant d_{0}, V_{i}(x) \leqslant M\right\}=0, \forall M>0,
$$

where $i=1,2$ and meas $(\cdot)$ denotes the Lebesgue measure in $\mathbb{R}^{\mathrm{N}}$.

This kind of hypotheses was first introduced by Bartsch and Wang [2, 24] in the study of a nonlinear Schrödinger equation. The hypotheses $\left(F_{1}\right)-\left(F_{2}\right)$ imply that $V_{i}(x)$ represents a potential well whose depth is controlled by $\lambda$ and $V_{i}(x)$ is called a steep potential well if $\lambda$ is large, where $(i=1,2)$.

Define

$$
\mathrm{N}_{\lambda}:=\left\{(\mathrm{u}, v) \in \mathrm{E}_{\lambda} \backslash\{(0,0)\}:\left\langle\mathrm{I}_{\lambda}^{\prime}(\mathrm{u}, v),(\mathrm{u}, v)\right\rangle=0\right\} .
$$

Then, $N_{\lambda}$ is a Nehari manifold associated to $I_{\lambda}$. We point out that the assumption $\left(F_{2}\right)$ is very weak even in dealing with the semilinear operator $-\Delta+(\lambda \alpha(x)+1) \mathrm{I}$ on $\mathbb{R}^{\mathrm{N}}$.

Now we state our main results.

Theorem 1.1. Under assumptions $\left(\mathrm{F}_{1}\right)-\left(\mathrm{F}_{2}\right)$, problem (1.1) has a least energy solution, i.e., a nontrivial solution $\left(u_{0}, v_{0}\right)$ satisfying $\mathrm{I}_{\lambda}\left(\mathrm{u}_{0}, v_{0}\right)=\inf _{\mathrm{N}_{\lambda}} \mathrm{I}_{\lambda}(\mathrm{u}, v)$. 
Theorem 1.2. Assume that $\left(u_{\lambda}, v_{\lambda}\right)$ is the ground state solution obtained by Theorem 1.1. Then $\left(u_{\lambda}, v_{\lambda}\right) \rightarrow$ $\left(\mathrm{u}_{0}, v_{0}\right)$ as $\lambda \rightarrow \infty$, and $\left(\mathrm{u}_{0}, v_{0}\right)$ is the nontrivial ground state solution of

$$
\begin{cases}\triangle^{2} u-\triangle u+u-\frac{1}{2} \triangle\left(u^{2}\right) u=\frac{p}{p+q}|u|^{p-2}|v|^{q} u, & \text { in } \mathbb{R}^{\mathrm{N}}, \\ \triangle^{2} v-\triangle v+v-\frac{1}{2} \triangle\left(v^{2}\right) v=\frac{q}{p+q}|u|^{p}|v|^{q-2} v, & \text { in } \mathbb{R}^{\mathrm{N}}\end{cases}
$$

Corollary 1.3. Under assumptions $\left(\mathrm{F}_{1}\right)-\left(\mathrm{F}_{2}\right)$, system (1.1) has at least one nontrivial solution. Moreover if $\left(u_{\lambda}, v_{\lambda}\right) \rightarrow\left(u_{0}, v_{0}\right)$ as $\lambda \rightarrow \infty$, and $\left(u_{0}, v_{0}\right)$ is the nontrivial solution of

$$
\begin{cases}\triangle^{2} u-\triangle u+u-\frac{1}{2} \triangle\left(u^{2}\right) u=\frac{p}{p+q}|u|^{p-2}|v|^{q} u, & \text { in } \mathbb{R}^{N}, \\ \triangle^{2} v-\triangle v+v-\frac{1}{2} \triangle\left(v^{2}\right) v=\frac{q}{p+q}|u|^{p}|v|^{q-2} v, & \text { in } \mathbb{R}^{N} .\end{cases}
$$

In this paper we make use of the following notations: $C$ will denote various positive constants; the strong (respectively weak) convergence is denoted by $\rightarrow$ (respectively $\rightarrow$ ); o(1) denotes o(1) $\rightarrow 0$ as $n \rightarrow \infty, B_{\rho}(0)$ denotes a ball centered at the origin with radius $\rho>0$.

The remainder of this paper is organized as follows. In Section 2, some preliminary results are presented. In Section 3, we give the proof of our main results.

\section{Variational setting and preliminaries} set

In this section, we give the variational setting for (1.1) and establish the compactness conditions. We

$$
E=\left\{(u, v) \in H^{2}\left(\mathbb{R}^{N}\right): \int_{\mathbb{R}^{N}} \alpha(x) u^{2} \mathrm{~d} x<\infty, \int_{\mathbb{R}^{N}} \beta(x) u^{2} \mathrm{~d} x<\infty\right\}
$$

be equipped with the inner product and norm

$$
\langle u, w\rangle=\int_{\mathbb{R}^{N}}(\triangle u \triangle w+\nabla u \nabla w+u w) d x, \quad\|u\|=\langle u, u\rangle^{\frac{1}{2}} .
$$

For $\lambda>0$, we also need the following inner product and norm

$$
\begin{array}{rlrl}
\langle\mathrm{u}, w\rangle_{\lambda} & =\int_{\mathbb{R}^{N}}\left(\triangle \mathrm{u} \triangle \boldsymbol{w}+\nabla u \nabla w+\mathrm{V}_{1}(\mathrm{x}) \mathrm{uw}\right) \mathrm{d} \mathrm{x}, & \|\mathrm{u}\|_{\lambda}=\langle\mathrm{u}, \mathrm{u}\rangle_{\lambda^{\prime}}^{\frac{1}{2}} \\
\langle v, \mathrm{~h}\rangle_{\lambda}=\int_{\mathbb{R}^{N}}\left(\triangle \mathrm{u} \triangle \mathrm{h}+\nabla \mathrm{u} \nabla \mathrm{h}+\mathrm{V}_{2}(\mathrm{x}) v \mathrm{~h}\right) \mathrm{d} \mathrm{x}, & \|v\|_{\lambda}=\langle v, v\rangle_{\lambda^{\prime}}^{\frac{1}{2}}
\end{array}
$$

with norm

$$
\|(u, v)\|_{\lambda}^{2}=\|u\|_{\lambda}^{2}+\|v\|_{\lambda}^{2} .
$$

It follows from $\left(F_{1}\right)-\left(F_{2}\right)$ and Poincaré's inequality that the embedding $E \hookrightarrow H^{2}\left(\mathbb{R}^{N}\right)$ is continuous.

Now we will work on Hilbert space E. A weak solution for problem (1.1) is a critical point of the following energy functional $I_{\lambda}$ defined on $E$ by

$$
\begin{aligned}
\mathrm{I}_{\lambda}(\mathrm{u}, v)= & \frac{1}{2} \int_{\mathbb{R}^{N}}|\Delta \mathrm{u}|^{2}+|\nabla \mathrm{u}|^{2}+\mathrm{V}_{1}(\mathrm{x}) \mathrm{u}^{2} \mathrm{~d} x+\frac{1}{2} \int_{\mathbb{R}^{N}}|\Delta v|^{2}+|\nabla v|^{2}+\mathrm{V}_{2}(\mathrm{x}) v^{2} \mathrm{~d} x \\
& +\frac{1}{2} \int_{\mathbb{R}^{N}}\left(\mathrm{u}^{2}|\nabla u|^{2}+v^{2}|\nabla v|^{2}\right) \mathrm{d} x-\frac{1}{p+\mathrm{q}} \int_{\mathbb{R}^{N}}|\mathrm{u}|^{p}|v|^{\mathrm{q}} \mathrm{d} x
\end{aligned}
$$

for all $(u, v) \in E$. By standard arguments, we see that $I(u, v)$ is a $C^{1}$ functional on the space E. Moreover $\mathrm{I}_{\lambda}^{\prime}(u, v)=0$ if and only if $(u, v)$ satisfies, for all $(\phi, \varphi) \in E$,

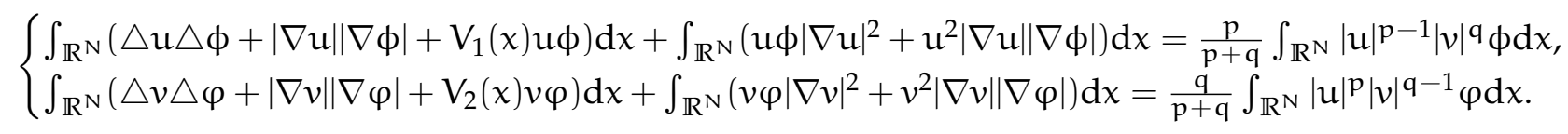


Recall that I satisfies the (PS) condition at the level $c \in R\left((P S)_{c}\right.$ condition for short) if any sequence $\left\{u_{n}\right\} \subset E_{\lambda}$ along with $\mathrm{I}\left(\mathrm{u}_{n}\right) \rightarrow c$ and $\mathrm{I}^{\prime}\left(\mathrm{u}_{\mathrm{n}}\right) \rightarrow 0$ as $\mathrm{n} \rightarrow \infty$ possesses a convergent subsequence. If I satisfies $(P S)_{c}$ condition for each $c \in R$, then we say that I satisfies the (PS) condition. In this paper, we will take $\mathrm{I}_{\lambda}=\mathrm{I}_{\lambda}(\mathrm{u}, v)$ and $\mathrm{E}_{\lambda}=\left(\mathrm{E},\|(\mathrm{u}, v)\|_{\lambda}\right)$.

Definition 2.1. A function $u$ is a ground state solution of problem (1.1), we mean that $u$ is such a solution which has the least energy among all nontrivial solutions of problem (1.1).

Lemma 2.2. Under the assumptions $\left(\mathrm{F}_{0}\right)$, the embedding $\mathrm{E}_{\lambda} \hookrightarrow \mathrm{L}^{\mathrm{s}}\left(\mathbb{R}^{\mathrm{N}}\right) \times \mathrm{L}^{\mathrm{s}}\left(\mathbb{R}^{\mathrm{N}}\right)$ is compact for $2 \leqslant \mathrm{~s}<2^{* *}$.

Proof. Using Lemma 3.4 in [34], the proofs is similar to Lemma 2.1 in [5], so we omit it here.

\section{Proof of main results}

In the following we show that the functional $\mathrm{I}_{\lambda}$ satisfies the mountain pass geometry.

Lemma 3.1. The functional $\mathrm{I}_{\lambda}$ satisfies the following conditions.

(i) There exists $\rho$ such that $\mathrm{I}_{\lambda}(u, v) \geqslant \alpha$ with $\|(u, v)\|_{\lambda}=\rho$.

(ii) There exists $\left(\mathrm{u}_{0}, v_{0}\right) \in \mathrm{B}_{\rho}(0)$ with $\mathrm{I}_{\lambda}\left(\mathrm{u}_{0}, v_{0}\right)<0$.

Proof. Using the Hölder inequality, we obtain

$$
\int_{\mathbb{R}^{N}}|u|^{p}|v|^{q} \mathrm{~d} x \leqslant\left(\int_{\mathbb{R}^{N}}|u|^{p+q} \mathrm{~d} x\right)^{\frac{p}{p+q}}\left(\int_{\mathbb{R}^{N}}|v|^{p+q} \mathrm{~d} x\right)^{\frac{q}{p+q}}
$$

According to the Hölder and Nirenberg inequalities for any $p+q \in\left[4,2^{* *}\right)$ there exists $C>0$, such that

$$
\begin{aligned}
& \int_{\mathbb{R}^{N}}|u|^{p+q} \mathrm{~d} x \leqslant\left(\int_{\mathbb{R}^{N}}|u|^{2} \mathrm{~d} x\right)^{\frac{[2 N-(p+q)(N-4)]}{8}}\left(\int_{\mathbb{R}^{N}}|u|^{2^{* *}} \mathrm{~d} x\right)^{\frac{(p+q-2)(N-4)}{8}} \\
& \leqslant C_{1}^{\frac{N(p+q-2)}{4}}\left(\int_{\mathbb{R}^{N}}|u|^{2} d x\right)^{\frac{[2 N-(p+q)(N-4)]}{8}}\left(\int_{\mathbb{R}^{N}}|\triangle u|^{2} d x\right)^{\frac{N(p+q-2)}{8}} \leqslant C\|(u, v)\|_{\lambda}^{p+q} .
\end{aligned}
$$

We denote $C=C_{1}^{\frac{N(p+q-2)}{4}}$. Similarly, we have

$$
\int_{\mathbb{R}^{N}}|u|^{p+q} d x \leqslant C\|(u, v)\|_{\lambda}^{p+q} .
$$

By (3.1), we obtain

$$
\begin{aligned}
\mathrm{I}_{\lambda}(\mathrm{u}, v) & =\frac{1}{2}\|(\mathrm{u}, v)\|_{\lambda}^{2}+\frac{1}{2} \int_{\mathbb{R}^{\mathrm{N}}}\left(\mathrm{u}^{2}|\nabla \mathrm{u}|^{2}+v^{2}|\nabla v|^{2}\right) \mathrm{d} x-\frac{1}{\mathrm{p}+\mathrm{q}} \int_{\mathbb{R}^{\mathrm{N}}}|\mathrm{u}|^{\mathrm{p}}|v|^{\mathrm{q}} \mathrm{d} x \\
& \geqslant \frac{1}{2}\|(\mathrm{u}, v)\|_{\lambda}^{2}-\frac{1}{\mathrm{p}+\mathrm{q}} \int_{\mathbb{R}^{N}}|\mathrm{u}|^{\mathrm{p}}|v|^{\mathrm{q}} \mathrm{d} x \geqslant \frac{1}{2}\|(\mathrm{u}, v)\|_{\lambda}^{2}-\mathrm{C}\|(\mathrm{u}, v)\|_{\lambda}^{\mathrm{p}+\mathrm{q}} .
\end{aligned}
$$

Hence, we can choose small enough $\rho>0$, so there exists $\alpha>0$ such that $I_{\lambda}(u, v) \geqslant \alpha$ with $\|(u, v)\|_{\lambda}=\rho$.

(ii) We see that for all $t \in \mathbb{R}$. We have

$$
\mathrm{I}_{\lambda}(\mathrm{tu}, \mathrm{t} v)=\frac{\mathrm{t}^{2}}{2}\|(\mathrm{u}, v)\|_{\lambda}^{2}+\frac{\mathrm{t}^{4}}{2} \int_{\mathbb{R}^{N}}\left(\mathrm{u}^{2}|\nabla u|^{2}+v^{2}|\nabla v|^{2}\right) \mathrm{d} x-\frac{\mathrm{t}^{\mathrm{p}+\mathrm{q}}}{\mathrm{p}+\mathrm{q}} \int_{\mathbb{R}^{N}}|\mathrm{u}|^{\mathrm{p}}|v|^{\mathrm{q}} \mathrm{d} x<0
$$

for $t>0$ large enough. Hence, we take $e=t^{*}\left(u_{0}, v_{0}\right)$ with some $t^{*}>0$ and (ii) follows.

Lemma 3.2. For any $(\mathrm{u}, v) \in \mathrm{E}_{\lambda} \backslash\{0\}$, there exists a unique $\mathrm{t}>0$ such that $(\mathrm{t} u, \mathrm{t} v) \in \mathrm{N}_{\lambda}$. Moreover, $\mathrm{I}_{\lambda}(\mathrm{tu}, \mathrm{t} v)=$ $\max _{\mathrm{t} \geqslant 0} \mathrm{I}_{\lambda}(\mathrm{tu}, \mathrm{t} v)$.

Proof. Let $(u, v) \in E_{\lambda} \backslash\{0\}$ be fixed and define the function $g(t)=I_{\lambda}(t u, t v)$ for $t \geqslant 0$. It is easy to obtain that $g(0)=0$, by Lemma 3.1, we have $g(t)>0$ for small $t>0$. Moreover, applying a similar argument as in the proof of Lemma 3.1, it is easy to see that $g(t)<0$ for large enough $t$. Hence, $\max _{t \geqslant 0} I_{\lambda}(t u, t v)$ 
exists and is achieved at $t>0$. Thus, we get $g^{\prime}(t)=0$, that is,

$$
\mathrm{I}_{\lambda}^{\prime}(\mathrm{tu}, \mathrm{t} v)=\mathrm{t}\|(\mathrm{u}, v)\|_{\lambda}^{2}-\mathrm{t}^{\mathrm{p}+\mathrm{q}-1} \int_{\mathbb{R}^{N}}|\mathrm{u}|^{\mathrm{p}}|v|^{\mathrm{q}} \mathrm{d} x+2 \mathrm{t}^{3} \int_{\mathbb{R}^{\mathrm{N}}}\left(\mathrm{u}^{2}|\nabla u|^{2}+v^{2}|\nabla v|^{2}\right) \mathrm{d} x=0,
$$

which implies $(t u, t v) \in N_{\lambda}$. So we obtain

$$
\int_{\mathbb{R}^{N}}\left(u^{2}|\nabla u|^{2}+v^{2}|\nabla v|^{2}\right) \mathrm{d} x=-\frac{\|(u, v)\|_{\lambda}^{2}}{2 t^{2}}+\frac{t^{p+q}}{2 t^{4}} \int_{\mathbb{R}^{N}}|u|^{p}|v|^{q} \mathrm{~d} x .
$$

By (3.2) and $4<p+q<2^{* *}$, we obtain that the right is strictly increasing about $t \geqslant 0$. It follows from (3.3) that there exists a unique $t_{0}>0$, such that $\left(t_{0} u, t_{0} v\right) \in N_{\lambda}$ and $I_{\lambda}\left(t_{0} u, t_{0} v\right)=\max _{t \geqslant 0} I_{\lambda}\left(t_{0} u, t_{0} v\right)$.

Lemma 3.3. Assume that $\left(\mathrm{F}_{1}\right)$ and $\left(\mathrm{F}_{2}\right)$ hold, then $\mathrm{I}_{\lambda}(\mathrm{u}, v)$ satisfies (PS) condition.

Proof. We first prove that $\left\{\left(u_{n}, v_{n}\right)\right\}$ is bounded in $E_{\lambda}$. We take $(u, v) \in E_{\lambda}$. So we obtain

$$
\begin{aligned}
c+o(1) & \geqslant I_{\lambda}\left(u_{n}, v_{n}\right)-\frac{1}{4}\left\langle I_{\lambda}^{\prime}\left(u_{n}, v_{n}\right),\left(u_{n}, v_{n}\right)\right\rangle \\
& =\frac{1}{4}\left\|\left(u_{n}, v_{n}\right)\right\|_{\lambda}^{2}+\left.\left(\frac{1}{4}-\frac{1}{p+q}\right) \int_{\mathbb{R}^{N}}\left|u_{n}\right|\right|^{p}\left|v_{n}\right|^{q} d x \geqslant \frac{1}{4}\left\|\left(u_{n}, v_{n}\right)\right\|_{\lambda}^{2},
\end{aligned}
$$

which implies that $\left\{\left(u_{n}, v_{n}\right)\right\}$ is bounded.

Finally, we show that $\left\{\left(u_{n}, v_{n}\right)\right\}$ possesses a strong convergent subsequence in $E_{\lambda}$. In fact, in view of the boundedness of $\left\{\left(u_{n}, v_{n}\right)\right\}$, without loss of generality, we may assume $\left(u_{n}, v_{n}\right) \rightarrow\left(u_{0}, v_{0}\right)$ as $n \rightarrow \infty$ for some $\left(u_{0}, v_{0}\right) \in E_{\lambda}$. Since $E_{\lambda} \hookrightarrow L^{s}\left(\mathbb{R}^{N}\right) \times L^{s}\left(\mathbb{R}^{N}\right)$ is compact for $s \in\left[2,2^{* *}\right),\left(u_{n}, v_{n}\right) \rightarrow\left(u_{0}, v_{0}\right)$ in $\mathrm{L}^{\mathrm{s}}\left(\mathbb{R}^{\mathrm{N}}\right) \times \mathrm{L}^{\mathrm{s}}\left(\mathbb{R}^{\mathrm{N}}\right)$ for any $\mathrm{s} \in\left[2,2^{* *}\right)$. We easily obtain

$$
\begin{aligned}
& \left\langle\left(I_{\lambda}^{\prime}\left(u_{n}, v_{n}\right)-I_{\lambda}^{\prime}(u, v)\right),\left(\left(u_{n}-u, v_{n}-v\right)\right)\right\rangle \\
& =\left\|\left(u_{n}-u\right),\left(v_{n}-v\right)\right\|_{\lambda}^{2}+\int_{\mathbb{R}^{N}}\left(u_{n}^{2} \nabla u_{n}-u^{2} \nabla u\right) \nabla\left(u_{n}-u\right) d x+\int_{\mathbb{R}^{N}}\left(v_{n}\left|\nabla v_{n}\right|^{2}-v|\nabla v|^{2}\right)\left(v_{n}-v\right) \mathrm{d} x \\
& \quad+\int_{\mathbb{R}^{N}}\left(v_{n}^{2} \nabla v_{n}-v^{2} \nabla v\right) \nabla\left(v_{n}-v\right) \mathrm{d} x+\int_{\mathbb{R}^{N}}\left(\left|u_{n}\right|^{p-1}\left|v_{n}\right|^{q}-|u|^{p-1}|v|^{q}\right)\left|u_{n}-u\right| d x \\
& \quad+\int_{\mathbb{R}^{N}}\left(u_{n}\left|\nabla u_{n}\right|^{2}-u|\nabla u|^{2}\right)\left(u_{n}-u\right) d x+\int_{\mathbb{R}^{N}}\left(\left|v_{n}\right|^{q-1}\left|u_{n}\right|^{p}-|v|^{q-1}|u|^{p}\right)\left|v_{n}-v\right| d x .
\end{aligned}
$$

It is clear that

$$
\left\langle\left(\mathrm{I}_{\lambda}^{\prime}\left(\mathrm{u}_{\mathrm{n}}, v_{\mathrm{n}}\right)-\mathrm{I}_{\lambda}^{\prime}(\mathrm{u}, v)\right),\left(\left(\mathrm{u}_{\mathrm{n}}-\mathrm{u}, v_{\mathrm{n}}-v\right)\right)\right\rangle \rightarrow 0 .
$$

By the Hölder's inequality we can get that

$$
\begin{aligned}
& \lim _{n \rightarrow \infty} \int_{\mathbb{R}^{N}}\left(\left|u_{n}\right|^{p-1}\left|v_{n}\right|^{q}-|u|^{p-1}|v| q\right)\left|u_{n}-u\right| d x \\
& \quad \leqslant C \lim _{n \rightarrow \infty} \int_{\mathbb{R}^{N}}\left|u_{n}-u\right|^{p}\left|v_{n}-v\right|^{q} d x \\
& \quad \leqslant C \lim _{n \rightarrow \infty}\left(\int_{\mathbb{R}^{N}}\left|u_{n}-u\right|^{p+q} d x\right)^{\frac{p}{p+q}}\left(\int_{\mathbb{R}^{N}}\left|v_{n}-v\right|^{p+q} d x\right)^{\frac{q}{p+q}} .
\end{aligned}
$$

Note that $u_{n} \rightarrow u$ in $L^{r}\left(\mathbb{R}^{N}\right)$ for $2<q<2^{* *}$. Since $4<p+q<22^{* *}$, we can choose $k \in(0,1)$ such that $p+q=k r+(1-k) 22^{* *}$, then

$$
\int_{\mathbb{R}^{N}}\left|u_{n}-u\right|^{p+q} d x \leqslant\left(\int_{\mathbb{R}^{N}}\left|u_{n}-u\right|^{q} d x\right)^{\frac{1}{k}}\left(\int_{\mathbb{R}^{N}}\left|u_{n}-u\right|^{22^{* *}} d x\right)^{\frac{1}{1-k}} \rightarrow 0 .
$$


Similarly, we have

$$
\begin{aligned}
& \lim _{n \rightarrow \infty} \int_{\mathbb{R}^{N}}\left(\left|v_{n}\right|^{q-1}\left|u_{n}\right|^{p}-|v|^{q-1}|u|^{p}\right)\left|v_{n}-v\right| d x \\
& \quad \leqslant \lim _{n \rightarrow \infty}\left(\int_{\mathbb{R}^{N}}\left|u_{n}-u\right|^{p+q} d x\right)^{\frac{p}{p+q}}\left(\int_{\mathbb{R}^{N}}\left|v_{n}-v\right|^{p+q} d x\right)^{\frac{q}{p+q}} \rightarrow 0 .
\end{aligned}
$$

Note that $n \leqslant 5$ and $6<2^{* *}$. Using Hölder's inequality and $u_{n} \rightarrow u$ in $L^{s}\left(R^{N}\right)$ for any $s \in\left[2,2^{* *}\right)$, we have

$$
\begin{aligned}
& \left|\int_{\mathbb{R}^{N}}\left(u_{n}\left|\nabla u_{n}\right|^{2}-u|\nabla u|^{2}\right)\left(u_{n}-u\right) d x\right| \leqslant \int_{\mathbb{R}^{N}}\left|u_{n}\right|\left|\nabla u_{n}\right|^{2}\left|u_{n}-u\right| d x+\int_{\mathbb{R}^{N}}|u||\nabla u|^{2}\left|u_{n}-u\right| d x \\
& \leqslant\left(\int_{\mathbb{R}^{N}}\left|u_{n}\right|^{6} \mathrm{~d} x\right)^{\frac{1}{6}}\left(\int_{\mathbb{R}^{N}}\left|\nabla u_{n}\right|^{3} \mathrm{~d} x\right)^{\frac{2}{3}}\left(\int_{\mathbb{R}^{N}}\left|u_{n}-u\right|^{6} \mathrm{~d} x\right)^{\frac{1}{6}} \\
& +\left(\int_{\mathbb{R}^{N}}|\mathfrak{u}|^{6} \mathrm{~d} x\right)^{\frac{1}{6}}\left(\int_{\mathbb{R}^{N}}|\nabla u|^{3} \mathrm{~d} x\right)^{\frac{2}{3}}\left(\int_{\mathbb{R}^{N}}\left|u_{n}-\mathfrak{u}\right|^{6} \mathrm{~d} x\right)^{\frac{1}{6}} \\
& \leqslant C\left\|u_{n}-u\right\|_{L^{6}} \rightarrow 0 \text {, as } n \rightarrow \infty \text {. }
\end{aligned}
$$

Here again

$$
\left|\int_{\mathbb{R}^{N}}\left(v_{n}\left|\nabla v_{n}\right|^{2}-v|\nabla v|^{2}\right)\left(v_{n}-v\right) \mathrm{d} x\right| \rightarrow 0
$$

Similarly we can also obtain

$$
\begin{aligned}
& \left|\int_{\mathbb{R}^{N}}\left(u_{n}^{2}-u^{2}\right) \nabla u_{n} \nabla\left(u_{n}-u\right) d x\right| \\
& \quad \leqslant \int_{\mathbb{R}^{N}}\left|u_{n}-u\right|\left|u_{n}+u\right| \nabla u_{n}|| \nabla\left(u_{n}-u\right) \mid d x \\
& \quad \leqslant\left(\int_{\mathbb{R}^{N}}\left|u_{n}-u\right|^{6}\right)^{\frac{1}{6}}\left(\int_{\mathbb{R}^{N}}\left|u_{n}+u\right|^{6}\right)^{\frac{1}{6}}\left(\int_{\mathbb{R}^{N}}\left|\nabla u_{n}\right|^{3}\right)^{\frac{1}{3}}\left(\int_{\mathbb{R}^{N}}\left|\nabla\left(u_{n}-u\right)\right|^{3}\right)^{\frac{1}{3}} \\
& \quad \leqslant C\left\|u_{n}-u\right\|_{L^{6}} \rightarrow 0, \text { as } n \rightarrow \infty .
\end{aligned}
$$

We also get

$$
\left|\int_{\mathbb{R}^{N}}\left(v_{\mathrm{n}}^{2}-v^{2}\right) \nabla v_{\mathrm{n}} \nabla\left(v_{\mathrm{n}}-v\right) \mathrm{d} x\right| \rightarrow 0 .
$$

Thus we obtain that $\left\|\left(u_{n}-u\right),\left(v_{n}-v\right)\right\|_{\lambda}^{2} \rightarrow 0$. This completes the proof.

We define

$$
c=\inf _{\eta \in \Gamma} \sup _{t \in[0,1]} I_{\lambda}(\eta(t)), \quad c^{*}=\inf _{(u, v) \in N_{\lambda}} I_{\lambda}(u, v), \quad c^{* *}=\inf _{(u, v) \in E_{\lambda} \backslash\{0\}} \sup _{t \geqslant 0} I_{\lambda}(t u, t v),
$$

where $u$ is given by Lemma 3.2. We also define

$$
\begin{aligned}
\widetilde{\gamma_{1}}(\mathrm{t}) & :=\mathrm{tu} \text { for } 0 \leqslant \mathrm{t} \leqslant 1, \widetilde{\gamma_{2}}(\mathrm{~s}):=\mathrm{s} v \text { for } 0 \leqslant s \leqslant 1, \quad \text { and } \widetilde{\gamma}(\mathrm{t}, \mathrm{s}):=\left(\widetilde{\gamma_{1}}(\mathrm{t}), \widetilde{\gamma_{2}}(\mathrm{~s})\right), \\
\Gamma & =\left\{\eta \in \mathrm{C}([0,1], X) \widetilde{\gamma}(0,0)=0, \mathrm{I}_{\lambda}(\widetilde{\gamma}(1,1)) \leqslant 0, \widetilde{\gamma}(1,1) \neq 0\right\} .
\end{aligned}
$$

Lemma 3.4. $c=c^{*}=c^{* *}$.

Proof. The proof is standard. We sketch it here for the completeness of the paper. It follows from Lemma 3.2 that $c^{*}=c^{* *}$. Note that for any $(u, v) \in E_{\lambda} \backslash\{0\}$, there exists $T>0$ large enough such that $\mathrm{I}_{\lambda}(\mathrm{tu}, \mathrm{sv})<0$. We may define a path $\widetilde{\gamma}(t, s)=(t u, s v)$, clearly, $\widetilde{\gamma} \in \Gamma$ and consequently, $c \leqslant c^{*}=c^{* *}$. Now, we show that 
$c^{*} \leqslant c$. By $(2.1)$ and Lemma 3.1,

$$
\begin{aligned}
\left\langle\mathrm{I}_{\lambda}^{\prime}(\mathrm{u}, v),(\mathrm{u}, v)\right\rangle & =\|(\mathrm{u}, v)\|_{\lambda}^{2}+2 \int_{\mathbb{R}^{N}}\left(\mathrm{u}^{2}|\nabla u|^{2}+v^{2}|\nabla v|^{2}\right) \mathrm{d} x-\int_{\mathbb{R}^{N}}|u|^{p}|v|^{\mathrm{q}} \mathrm{d} x \\
& \geqslant\|(u, v)\|_{\lambda}^{2}-\mathrm{C}\|(\mathrm{u}, v)\|_{\lambda}^{p+q} .
\end{aligned}
$$

Since, $\|u\|_{\lambda}^{2}+\|v\|_{\lambda}^{2} \rightarrow 0$, if $\|(u, v)\|_{\lambda} \rightarrow 0$, it follows from (3.5) that for nonzero $(u, v)$ with small enough $\|(u, v)\|_{\lambda},\left\langle\mathrm{I}_{\lambda}^{\prime}(u, v),(u, v)\right\rangle>0$. Thus from Lemma 3.1, for small enough $\|(u, v)\|_{\lambda}$, we get

$$
\|u\|_{\lambda}^{2}+\|v\|_{\lambda}^{2}+2 \int_{\mathbb{R}^{N}}\left(u^{2}|\nabla u|^{2}+v^{2}|\nabla v|^{2}\right) \mathrm{d} x>\frac{1}{2} \int_{\mathbb{R}^{N}}|u|^{p}|v|^{\mathrm{q}} \mathrm{d} x
$$

In fact, any path $\widetilde{\gamma}(t, s)$ in $\Gamma$ crosses $N_{\lambda}$. Otherwise, by the continuity of $\widetilde{\gamma}(t, s)$, inequality (3.6) still holds true for each nonzero $\widetilde{\gamma}(t, s),(t, s) \in[0,1] \times[0,1]$. Hence

$$
\begin{aligned}
\mathrm{I}_{\lambda}(\widetilde{\gamma}(1,1))= & \frac{1}{2}\left\|\left(\widetilde{\gamma_{1}}(1), \widetilde{\gamma_{2}}(1)\right)\right\|^{2}+\frac{1}{2} \int_{\mathbb{R}^{\mathrm{N}}}\left(\widetilde{\gamma_{1}}(1)^{2}\left|\nabla \widetilde{\gamma_{1}}(1)\right|^{2}+\widetilde{\gamma_{2}}(1)^{2}\left|\nabla \widetilde{\gamma_{2}}(1)\right|^{2}\right) \mathrm{d} x \\
& -\left.\left.\frac{1}{\mathrm{p}+\mathrm{q}} \int_{\mathbb{R}^{N}} \widetilde{\gamma_{1}}(1)\right|^{\mathrm{p}} \widetilde{\gamma_{2}}(1)\right|^{\mathrm{q}} \mathrm{d} x>\left(\frac{1}{4}-\frac{1}{\mathrm{p}+\mathrm{q}}\right) \int_{\mathbb{R}^{\mathrm{N}}}\left|\widetilde{\gamma_{1}}(1)\right|^{p}\left|\widetilde{\gamma_{2}}(1)\right|^{\mathrm{q}} \mathrm{d} x>0,
\end{aligned}
$$

which contradict the definition of $\widetilde{\gamma}(1,1)$. Thus, every path $\widetilde{\gamma}(t, s) \in \Gamma$ crosses $N_{\lambda}$ and consequently, $c^{*} \leqslant c$. This completes the proof.

Lemma 3.5. The critical points of the functional $\mathrm{I}_{\lambda}$ on $\mathrm{N}_{\lambda}$ are critical points of $\mathrm{I}_{\lambda}$ in $\mathrm{E}_{\lambda}$.

Proof. Let $\left\{\left(u_{n}, v_{n}\right)\right\} \subset N_{\lambda}$ be such that $I_{\lambda}\left(u_{n}, v_{n}\right) \rightarrow c$ and $I_{\lambda}^{\prime}\left(u_{n}, v_{n}\right) \rightarrow 0$. Then there exists $\mu_{n} \subset \mathbb{R}$ such that $\mathrm{I}_{\lambda}^{\prime}\left(u_{n}, v_{n}\right)=\mu_{n}{\widetilde{I^{\prime}}}_{\lambda}\left(u_{n}, v_{n}\right)+o(1)$, where

$$
\widetilde{\mathrm{I}}_{\lambda}(\mathrm{u}, v):=\|(\mathrm{u}, v)\|_{\lambda}^{2}+2 \int_{\mathbb{R}^{N}}\left(\mathrm{u}^{2}|\nabla u|^{2}+v^{2}|\nabla v|^{2}\right) \mathrm{d} x-\int_{\mathbb{R}^{N}}|u|^{p}|v|^{\mathrm{q}} \mathrm{d} x
$$

We denote that $\widetilde{\mathrm{I}}_{\lambda}(u, v)=\left\langle\mathrm{I}_{\lambda}^{\prime}(u, v),(u, v)\right\rangle$, thus

$$
\begin{aligned}
\left\langle{\widetilde{\mathrm{I}^{\prime}}}_{\lambda}(\mathrm{u}, v),(\mathrm{u}, v)\right\rangle & =2\|(\mathrm{u}, v)\|_{\lambda}^{2}-(\mathrm{p}+\mathrm{q}) \int_{\mathbb{R}^{N}}|\mathrm{u}|^{\mathrm{p}}|v|^{\mathrm{q}} \mathrm{d} x+4 \int_{\mathbb{R}^{N}}\left(\mathrm{u}^{2}|\nabla u|^{2}+v^{2}|\nabla v|^{2}\right) \mathrm{d} x \\
& \leqslant-(\mathrm{p}+\mathrm{q}-2) \int_{\mathbb{R}^{N}}|u|^{\mathrm{p}}|v|^{\mathrm{q}} \mathrm{d} x .
\end{aligned}
$$

If $(u, v) \in \mathrm{N}_{\lambda}$, since $(u, v) \neq 0$, then $\left\langle{\widetilde{\mathrm{I}^{\prime}}}_{\lambda}(\mathrm{u}, v),(\mathrm{u}, v)\right\rangle<0$ and hence ${\widetilde{\mathrm{I}^{\prime}}}_{\lambda}(\mathrm{u}, v) \neq 0$ on $\mathrm{N}_{\lambda}$. Let $\mathrm{J}_{\lambda}:=\mathrm{I}_{\lambda} \mid \mathrm{N}_{\lambda}$, then we have

$$
\mathrm{J}_{\lambda}^{\prime}(\mathrm{u}, v)=\mathrm{I}_{\lambda}^{\prime}(\mathrm{u}, v)-\frac{\left\langle\mathrm{I}_{\lambda}^{\prime}(\mathrm{u}, v),(\mathrm{u}, v)\right\rangle}{\left\|{\widetilde{\mathrm{I}^{\prime}}}_{\lambda}(\mathrm{u}, v)\right\|^{2}}{\widetilde{\mathrm{I}^{\prime}}}_{\lambda}(\mathrm{u}, v),
$$

and

$$
\left\langle\mathrm{J}_{\lambda}^{\prime}(\mathrm{u}), \mathrm{u}\right\rangle=\left\langle\mathrm{I}_{\lambda}^{\prime}(\mathrm{u}, v),(\mathrm{u}, v)\right\rangle\left(1-\frac{\left\langle\widetilde{\mathrm{I}}_{\lambda}^{\prime}(\mathrm{u}, v),(\mathrm{u}, v)\right\rangle}{\left\|{\widetilde{\mathrm{I}^{\prime}}}_{\lambda}(\mathrm{u}, v)\right\|^{2}}\right) .
$$

So $J_{\lambda}^{\prime}(u, v)=0$ if and only if $I^{\prime}(u, v)=0$.

Proposition 3.6. Assume that $\mathrm{C}_{0}$ is fixed, then for all $\varepsilon>0$, there exist $\Gamma_{\varepsilon}, \mathrm{R}_{\varepsilon}>0$ such that if $\left\{\left(u_{n}, v_{n}\right)\right\}$ is a (PS $)_{c}$ sequence of $\mathrm{I}_{\lambda}(\mathrm{u}, v)$ with $\lambda>\Gamma_{\varepsilon}, \mathrm{c} \leqslant \mathrm{C}_{0}$, then

$$
\int_{\bar{\Omega}_{R_{\varepsilon}}^{c}}\left|u_{n}\right|^{p}\left|v_{n}\right|^{q} \mathrm{~d} x \leqslant \varepsilon \text { for } 4<p+q<22^{* *},
$$

where $\bar{\Omega}_{\mathbb{R}_{\varepsilon}}^{c}=\left\{x \in \mathbb{R}^{N} \| x \mid \geqslant R_{\varepsilon}\right\}$. 
Proof. For $\forall \mathrm{R}>0$, we set

$$
\Omega(R):=\left\{x \in \mathbb{R}^{N}|| x \mid \geqslant R, \alpha(x) \geqslant M_{1}\right\}, \bar{\Omega}(R):=\left\{x \in \mathbb{R}^{N} \| x \mid \geqslant R, \alpha(x) \leqslant M_{1}\right\} .
$$

Then

$$
\begin{aligned}
\int_{\Omega(R)} u_{n}^{2} d x & \leqslant \frac{1}{\lambda M_{1}} \int_{\Omega(R)} \lambda \alpha(x) u_{n}^{2} d x \\
& \leqslant \frac{1}{\lambda M_{1}} \int_{\Omega(R)}\left(\left|\Delta u_{n}\right|^{2}+\left|\nabla u_{n}\right|^{2}+\lambda \alpha(x) u_{n}^{2}\right) d x \\
& \leqslant \frac{1}{\lambda M_{1}}\left\|\left(u_{n}, v_{n}\right)\right\|_{\lambda,}^{2} \quad\left(\left\|\left(u_{n}, v_{n}\right)\right\|_{\lambda} \text { is bounded }\right) \\
& \rightarrow 0, \text { as } \lambda \rightarrow \infty, \\
\int_{\bar{\Omega}(R)} u_{n}^{2} d x & \leqslant\left[\int_{R^{N}}\left(u_{n}^{2}\right)^{p} d x\right]^{\frac{1}{p}} m(\bar{\Omega}(R))^{\frac{p-1}{p}},\left(2<p<2^{* *}\right) \\
& \leqslant\left(\int_{\mathbb{R}^{N}} u_{n}^{2} d x\right)^{\frac{\theta}{2}}\left(\int_{\mathbb{R}^{N}} u_{n}^{* *} d x\right)^{\frac{1-\theta}{22^{* *}}} m(\bar{\Omega}(R))^{\frac{p-1}{p}},(0<\theta<1) \\
& \leqslant C\left\|\left(u_{n}, v_{n}\right)\right\|_{\lambda}^{\theta}\left\|\left(u_{n}, v_{n}\right)\right\|_{\lambda}^{1-\theta} m(\bar{\Omega}(R))^{\frac{p-1}{p}} \\
& \leqslant C m(\bar{\Omega}(R))^{\frac{p-1}{p}} \rightarrow 0, \text { as } R \rightarrow \infty .
\end{aligned}
$$

Applying the Gagliardo-Nirenberg inequality, we obtain

$$
\begin{aligned}
\int_{\bar{\Omega}_{R}^{c}}\left|u_{n}\right|^{p+q} \mathrm{~d} x & \leqslant C\left(\int_{\bar{\Omega}_{R}^{c}}\left|\Delta u_{n}\right|^{2} d x\right)^{\frac{(p+q) \theta}{2}}\left(\int_{\bar{\Omega}_{R}^{c}}\left|u_{n}\right|^{2} d x\right)^{\frac{(p+q)(1-\theta)}{2}},\left(\theta=\frac{N(p+q-2)}{2(p+q)}\right) \\
& \leqslant C\left\|\left(u_{n}, v_{n}\right)\right\|_{\lambda}^{(p+q) \theta}\left(\int_{\Omega(R)}\left|u_{n}\right|^{2} d x+\int_{\bar{\Omega}_{R}^{c}}\left|u_{n}\right|^{2} d x\right)^{\frac{(p+q)(1-\theta)}{2}} \\
& \leqslant C\left(\int_{\Omega(R)}\left|u_{n}\right|^{2} d x+\int_{\bar{\Omega}_{R}^{c}}\left|u_{n}\right|^{2} d x\right)^{\frac{(p+q)(1-\theta)}{2}} \\
& \rightarrow 0 \text { as } \lambda, R \rightarrow \infty(\text { by (3.7) and (3.8)). }
\end{aligned}
$$

Similarly, we obtain $\int_{\bar{\Omega}_{R}^{c}}\left|v_{n}\right|^{p+q} d x \leqslant \varepsilon$ for $\lambda, R \rightarrow \infty$. Finally, using the Hölder Inequality, we obtain

$$
\lim _{n \rightarrow \infty} \sup \int_{\bar{\Omega}_{R_{\varepsilon}}^{c}}\left|u_{n}\right|^{p}\left|v_{n}\right|^{q} d x \leqslant\left(\int_{\bar{\Omega}_{R_{\varepsilon}}}\left|u_{n}\right|^{p+q} d x\right)^{\frac{p}{p+q}}\left(\int_{\bar{\Omega}_{R_{\varepsilon}}^{c}}\left|v_{n}\right|^{p+q} d x\right)^{\frac{q}{p+q}} \leqslant \varepsilon .
$$

This completes the proof.

Proof of Theorem 1.1. We only need to prove that $c$ is achieved at some $u \in N_{\lambda}$ from Lemmas 3.4 and 3.5. Letting $\left\{\left(u_{n}, v_{n}\right)\right\} \subset E_{\lambda} \cap N_{\lambda}$ be a minimizing sequence of $I_{\lambda}$, then we have

$$
c+1 \geqslant I_{\lambda}\left(u_{n}, v_{n}\right)-\frac{1}{4}\left\langle I_{\lambda}^{\prime}\left(u_{n}, v_{n}\right),\left(u_{n}, v_{n}\right)\right\rangle=\frac{1}{4}\|(u, v)\|_{\lambda}^{2}+\left(\frac{1}{4}-\frac{1}{p+q}\right) \int_{\mathbb{R}^{N}}\left|u_{n}\right|^{p}\left|v_{n}\right|^{q} d x \geqslant \frac{1}{4}\|(u, v)\|_{\lambda}^{2} .
$$

Therefore, $\left\{\left(u_{n}, v_{n}\right)\right\}$ is bounded in $E_{\lambda} \cap N_{\lambda}$.

Recall the compact embedding

$$
E_{\lambda} \hookrightarrow \hookrightarrow L^{s}\left(\mathbb{R}^{N}\right) \times L^{s}\left(\mathbb{R}^{N}\right), \quad s \in\left[2,2^{* *}\right) .
$$


Thus, going if necessary to a subsequence, we may assume that there exists a function $u \in E_{\lambda}$ such that

$$
\left\{\begin{array}{l}
\left(u_{n}, v_{n}\right) \rightarrow(u, v) \text { in } E_{\lambda} \\
\left(u_{n}, v_{n}\right) \rightarrow(u, v) \text { in } L^{s}\left(\mathbb{R}^{N}\right) \times L^{s}\left(\mathbb{R}^{N}\right), \forall s \in\left[2,2^{* *}\right), \\
\left(u_{n}, v_{n}\right) \rightarrow(u, v) \text { a.e. on } \mathbb{R}^{N} \times \mathbb{R}^{N}
\end{array}\right.
$$

By Fatou Lemma,

$$
\begin{aligned}
c & =\lim _{n \rightarrow \infty}\left(I_{\lambda}\left(u_{n}, v_{n}\right)-\frac{1}{4}\left\langle I^{\prime}\left(u_{n}, v_{n}\right),\left(u_{n}, v_{n}\right)\right\rangle\right) \\
& \geqslant \liminf _{n \rightarrow \infty}\left(I_{\lambda}\left(u_{n}, v_{n}\right)-\frac{1}{4}\left\langle I^{\prime}\left(u_{n}, v_{n}\right),\left(u_{n}, v_{n}\right)\right\rangle\right) \\
& =\frac{1}{4}\|(u, v)\|_{\lambda}^{2}+\left(\frac{1}{4}-\frac{1}{p+q}\right) \int_{\mathbb{R}^{N}}\left(|u|^{p}|v|^{q}\right) d x=I_{\lambda}(u, v)-\frac{1}{4}\left\langle I^{\prime}(u, v),(u, v)\right\rangle=c .
\end{aligned}
$$

This implies that $I_{\lambda}(u, v)=c$. Hence $u$ is the problem of ground state solution.

Now, we prove that $(u, v) \neq(0,0)$.

$$
\begin{aligned}
\mathrm{I}_{\lambda}\left(\mathrm{u}_{n}, v_{n}\right)-\frac{1}{2}\left\langle\mathrm{I}_{\lambda}^{\prime}\left(\mathrm{u}_{n}, v_{n}\right),\left(\mathrm{u}_{n}, v_{n}\right)\right\rangle & =-\frac{1}{2} \int_{\mathbb{R}^{N}}\left(\left|\nabla u_{n}\right|^{2} u_{n}^{2}+\left|\nabla v_{n}\right|^{2} v_{n}^{2}\right) \mathrm{d} x+\left(\frac{1}{2}-\frac{1}{p+q}\right) \int_{\mathbb{R}^{N}}\left|u_{n}\right|^{p}\left|v_{n}\right|^{q} \mathrm{~d} x \\
& =c+o(1) .
\end{aligned}
$$

It deduces that

$$
\left(\frac{1}{2}-\frac{1}{p+q}\right) \int_{\mathbb{R}^{N}}\left|u_{n}\right|^{p}\left|v_{n}\right|^{q} \mathrm{~d} x \geqslant c+o(1) .
$$

Let $\varepsilon_{0}=\frac{1}{4} c_{0} \leqslant \frac{1}{4} \mathrm{c}$, since $v_{\mathrm{n}} \rightarrow v$ strongly in $\mathrm{L}^{\mathrm{r}}\left(\Omega_{\mathrm{R}}\right)$, for any $\mathrm{R}>0$ and $2<\mathrm{r}<2^{* *}$, by Proposition 3.6, there exists $\Gamma_{0}>0, R_{0}>0$ such that for $\lambda \geqslant \Gamma_{0}, R \geqslant R_{0}$,

$$
\left(\frac{1}{2}-\frac{1}{p+q}\right) \int_{\bar{\Omega}_{R}^{c}}\left|u_{n}\right| p\left|v_{n}\right|^{q} \mathrm{~d} x<\varepsilon_{0}
$$

thus

$$
\int_{\Omega_{R}}|u|^{p}|v|^{q} \mathrm{~d} x \geqslant \frac{1}{4} c>0
$$

which implies that $(u, v) \neq(0,0)$. This completes the proof.

Proof of Theorem 1.2. For any sequence $0<\lambda_{n} \rightarrow+\infty$, as $n \rightarrow+\infty$, let $\left(u_{n}, v_{n}\right):=\left(u_{\lambda_{n}}, v_{\lambda_{n}}\right)$ be the critical points of $I_{\lambda_{n}}(u, v)$ in Theorem 1.1. First, we show that $\left\{\left(u_{n}, v_{n}\right)\right\}$ is bounded. Since $\left(u_{n}, v_{n}\right)$ is the critical points of $\mathrm{I}_{\lambda_{n}}$, we have

$$
\begin{aligned}
c+o(1) & =I_{\lambda_{n}}\left(u_{n}, v_{n}\right) \\
& =\frac{1}{2}\left\|\left(u_{n}, v_{n}\right)\right\|_{\lambda_{n}}^{2}+\frac{1}{2} \int_{\mathbb{R}^{N}}\left|u_{n}\right|^{2}\left|\nabla u_{n}\right|^{2} \mathrm{~d} x+\frac{1}{2} \int_{\mathbb{R}^{N}}\left|v_{n}\right|^{2}\left|\nabla v_{n}\right|^{2} \mathrm{~d} x-\frac{1}{p+q} \int_{\mathbb{R}^{N}}\left|u_{n}\right|^{p}\left|v_{n}\right|^{q} \mathrm{~d} x \\
& \geqslant \frac{1}{4}\left\|\left(u_{n}, v_{n}\right)\right\|_{\lambda_{n}}^{2}
\end{aligned}
$$

which deduces that $\left\|\left(u_{n}, v_{n}\right)\right\|_{\lambda_{n}} \leqslant 2 c^{\frac{1}{2}}$, that is, $\left\|\left(u_{n}, v_{n}\right)\right\|_{\lambda_{n}}$ is bounded in $E_{\lambda_{n}}$. Therefore, we may assume the $\left(u_{n}, v_{n}\right) \rightarrow\left(u_{0}, v_{0}\right)$ weakly in $E_{\lambda_{n}}$ and $\left(u_{n}, v_{n}\right) \rightarrow\left(u_{0}, v_{0}\right)$ strongly in $L_{\text {loc }}^{r}\left(\mathbb{R}^{N}\right) \times L_{\text {loc }}^{r}\left(\mathbb{R}^{N}\right)$ for $2 \leqslant r<22^{* *}$. By Fatou's Lemma, we have

$$
\begin{aligned}
\int_{\mathbb{R}^{N}} \alpha(x) u_{0}^{2} \mathrm{~d} x+\int_{\mathbb{R}^{N}} \beta(x) v_{0}^{2} \mathrm{~d} x & \leqslant \lim _{n \rightarrow \infty} \inf \int_{\mathbb{R}^{N}} \alpha(x) u_{n}^{2} \mathrm{~d} x+\lim _{n \rightarrow \infty} \inf \int_{\mathbb{R}^{N}} \beta(x) v_{n}^{2} \mathrm{~d} x \\
& \leqslant \lim _{n \rightarrow \infty} \inf \frac{\left\|\left(u_{n}, v_{n}\right)\right\|_{\lambda_{n}}^{2}}{\lambda_{n}}=0
\end{aligned}
$$


which implies that $\left(u_{0}, v_{0}\right)=(0,0)$, a.e. in $\mathbb{R}^{\mathrm{N}} \backslash\left\{\alpha^{-1}(0) \cup \beta^{-1}(0)\right\}$ and $\left(u_{0}, v_{0}\right) \in H^{2}(\Omega) \cap H_{0}^{1}(\Omega)$. Now for any $(\varphi, \phi) \in \mathrm{C}_{0}^{\infty}\left(\mathbb{R}^{N}\right)$, since $\left\langle\mathrm{I}_{\lambda_{n}}^{\prime}\left(u_{n}, v_{n}\right),(\varphi, \phi)\right\rangle=0$, it is easy to check that

$$
\begin{aligned}
\int_{\mathbb{R}^{N}} & \left(\Delta \mathfrak{u}_{0} \Delta \varphi+\Delta v_{0} \Delta \phi+\nabla u_{0} \nabla \varphi+\nabla v_{0} \nabla \phi+v_{1}(x) u_{0} \varphi+v_{2}(x) v_{0} \phi\right) \mathrm{d} x \\
& +\int_{\mathbb{R}^{N}}\left(u_{0} \varphi\left|\nabla u_{0}\right|^{2}+u_{0}^{2}\left|\nabla u_{0}\right||\nabla \varphi|\right) \mathrm{d} x+\int_{\mathbb{R}^{N}}\left(v_{0} \phi\left|\nabla v_{0}\right|^{2}+v_{0}^{2}\left|\nabla v_{0}\right||\nabla \phi|\right) \mathrm{d} x \\
= & \frac{q}{p+q} \int_{\mathbb{R}^{N}}\left|v_{0}\right|^{q-1}\left|\mathfrak{u}_{0}\right|^{p} \phi d x+\frac{p}{p+q} \int_{\mathbb{R}^{N}}\left|\mathfrak{u}_{0}\right|^{p-1} v_{0}^{q} \varphi d x
\end{aligned}
$$

that is, $\left(u_{0}, v_{0}\right)$ is a weak of solution of (1.2) by that density of $C_{0}^{\infty}(\Omega)$ in $H^{2}(\Omega) \cap H_{0}^{1}(\Omega)$. Now we show that $u_{n} \rightarrow u_{0}, v_{n} \rightarrow v_{0}$ in $L^{r}\left(\mathbb{R}^{N}\right) \times L^{r}\left(\mathbb{R}^{N}\right)$ for $2 \leqslant r<22^{* *}$. Otherwise, by Lions vanishing Lemma $[18,25]$, there exist $\delta>0, R_{0}>0$ and $x_{n}, \overline{x_{n}} \in \mathbb{R}^{N}$, such that

$$
\int_{B\left(x_{n}, R_{0}\right)}\left(u_{n}-u_{0}\right)^{2} d x \geqslant \delta, \quad \int_{B\left(\overline{x_{n}}, R_{0}\right)}\left(v_{n}-v_{0}\right)^{2} d x \geqslant \delta .
$$

Moreover, $x_{n} \rightarrow \infty$, hence meas $\left(B\left(x_{n}, R_{0}\right) \cap\left\{x \in \mathbb{R}^{N}, \alpha(x)<M_{1}\right\}\right) \rightarrow 0$ and meas $\left(B\left(\overline{x_{n}}, R_{0}\right) \cap\{x \in\right.$ $\left.\left.\mathbb{R}^{N}, \beta(x)<M_{2}\right\}\right) \rightarrow 0$. By the Hölder inequality, we have

$$
\int_{B\left(x_{n}, R_{0}\right) \cap\left\{\alpha(x)<M_{1}\right\}}\left(u_{n}-u_{0}\right)^{2} d x \rightarrow 0, \quad \int_{B\left(\overline{x_{n}}, R_{0}\right) \cap\left\{\beta(x)<M_{2}\right\}}\left(v_{n}-v_{0}\right)^{2} d x \rightarrow 0 .
$$

Consequently

$$
\begin{aligned}
& \int_{\mathbb{R}^{N}}\left(\left|\triangle u_{n}\right|^{2}+\left|\Delta v_{n}\right|^{2}+\left|\nabla u_{n}\right|^{2}+\left|\nabla v_{n}\right|^{2}+\lambda_{n} \alpha(x)(x) u_{n}+\lambda_{n} \beta(x) v_{n}\right) \mathrm{d} x \\
& \geqslant \lambda_{n} M_{1} \int_{B\left(x_{n}, R_{0}\right) \cap\left\{\alpha(x) \geqslant M_{1}\right\}}\left|u_{n}\right|^{2} d x+\lambda_{n} M_{2} \int_{B\left(\overline{x_{n}}, R_{0}\right) \cap\left\{\beta \geqslant M_{2}\right\}}\left|v_{n}\right|^{2} d x \\
& \geqslant \lambda_{n} M_{1} \int_{B\left(x_{n}, R_{0}\right) \cap\left\{\alpha(x) \geqslant M_{1}\right\}}\left(u_{n}-u_{0}\right)^{2} d x+\lambda_{n} M_{2} \int_{B\left(\overline{x_{n}}, R_{0}\right) \cap\left\{\beta(x) \geqslant M_{2}\right\}}\left(v_{n}-v_{0}\right)^{2} d x \\
& =\lambda_{n} M_{1} \int_{B\left(x_{n}, R_{0}\right)}\left(u_{n}-u_{0}\right)^{2} d x-\lambda_{n} M_{1} \int_{B\left(x_{n}, R_{0}\right) \cap\left\{\alpha(x)<M_{1}\right\}}\left(u_{n}-u_{0}\right)^{2} d x+o(1) \\
& +\lambda_{n} M_{2} \int_{B\left(\overline{x_{n}}, R_{0}\right)}\left(v_{n}-v_{0}\right)^{2} \mathrm{~d} x-\lambda_{n} M_{2} \int_{B\left(\overline{x_{n}}, R_{0}\right) \cap\left\{\beta(x)<M_{2}\right\}}\left(v_{n}-v_{0}\right)^{2} \mathrm{~d} x+o(1) \\
& \rightarrow \infty \text {, as } \mathrm{n} \rightarrow \infty \text {, }
\end{aligned}
$$

which contradicts to (3.9). In the above proof, we have used the facts that $\left|B\left(x_{n}, R_{0}\right) \cap\left\{\alpha(x)<M_{1}\right\}\right| \rightarrow 0$, $\left|\mathrm{B}\left(\overline{x_{n}}, \mathrm{R}_{0}\right) \cap\left\{\beta(x)<M_{2}\right\}\right| \rightarrow 0$ as $\mathrm{n} \rightarrow \infty$ and also that the $\mathrm{L}^{2}$-norm of $\left\{\left(u_{n}, v_{n}\right)\right\}$ is bounded. Therefore, $u_{n} \rightarrow v_{0}, v_{n} \rightarrow v_{0}$ in $\mathrm{L}^{r}\left(\mathbb{R}^{N}\right) \times \mathrm{L}^{\mathrm{r}}\left(\mathbb{R}^{\mathrm{N}}\right)$ for $2 \leqslant \mathrm{r}<22^{* *}$.

In the following, we show that $\left(u_{n}, v_{n}\right) \rightarrow\left(u_{0}, v_{0}\right)$ in $H^{2}\left(\mathbb{R}^{N}\right) \times H^{2}\left(\mathbb{R}^{N}\right)$. Since

$$
\left\langle\mathrm{I}_{\lambda_{n}}^{\prime}\left(u_{n}, v_{n}\right),\left(u_{n}, v_{n}\right)\right\rangle=\left\langle I_{\lambda_{n}}^{\prime}\left(u_{n}, v_{n}\right),\left(u_{0}, v_{0}\right)\right\rangle=0,
$$

we have

$$
\begin{aligned}
& \left\|\left(u_{n}, v_{n}\right)\right\|_{\lambda_{n}}^{2}+2 \int_{\mathbb{R}^{N}} u_{n}^{2}\left|\nabla u_{n}\right|^{2} \mathrm{~d} x+2 \int_{\mathbb{R}^{N}} v_{n}^{2}\left|\nabla v_{n}\right|^{2} \mathrm{~d} x \\
& =\frac{p}{p+q} \int_{\mathbb{R}^{N}}\left|u_{n}\right|^{p}\left|v_{n}\right|^{\mathrm{q}} \mathrm{d} x+\frac{\mathrm{q}}{p+q} \int_{\mathbb{R}^{N}}\left|v_{n}\right|^{\mathrm{q}}\left|\mathrm{u}_{n}\right|^{\mathrm{p}} \mathrm{d} x
\end{aligned}
$$


and

$$
\begin{gathered}
\left\langle\left(u_{n}, v_{n}\right),\left(u_{0}, v_{0}\right)\right\rangle_{\lambda_{n}}+\int_{\mathbb{R}^{N}}\left(u_{n} u_{0}\left|\nabla u_{n}\right|^{2}+u_{n}^{2}\left|\nabla u_{n}\right|\left|\nabla u_{0}\right|\right) d x+\int_{\mathbb{R}^{N}}\left(v_{n} v_{0}\left|\nabla v_{n}\right|^{2}+v_{n}^{2}|\nabla v|\left|\nabla v_{0}\right|\right) d x \\
=\frac{p}{p+q} \int_{\mathbb{R}^{N}}\left|u_{n}\right|^{p-1}\left|v_{n}\right|^{q} u_{0} d x+\frac{q}{p+q} \int_{\mathbb{R}^{N}}\left|v_{n}\right|^{q-1}\left|u_{n}\right|^{p} v_{0} d x .
\end{gathered}
$$

It is easy to verify that

$$
\begin{aligned}
& \left|\int_{\mathbb{R}^{N}}\left(\left|u_{n}\right|^{p-1}\left|v_{n}\right|^{q} u_{n}-\left|u_{n}\right|^{p-1}\left|v_{n}\right|^{q} u_{0}\right) \mathrm{d} x\right| \rightarrow 0, \\
& \left.\left|\int_{\mathbb{R}^{N}}\left(\left|v_{n}\right|^{q-1}\left|u_{n}\right|^{p} v_{n}-\mid v_{n}\right)\right|^{q-1}\left|u_{n}\right|^{p} v_{0}\right) \mathrm{d} x \mid \rightarrow 0 .
\end{aligned}
$$

In fact for (3.12) similar to the proof of (3.4). Since

$$
\begin{aligned}
\left.\left|\int_{\mathbb{R}^{N}}\right| u_{n}|| \nabla u_{n}\right|^{2}\left(u_{n}-u_{0}\right) \mathrm{d} x \mid & \leqslant\left(\int_{\mathbb{R}^{N}}\left|u_{n}\right|^{6} \mathrm{~d} x\right)^{\frac{1}{6}}\left(\int_{\mathbb{R}^{N}}\left|\nabla u_{n}\right|^{3} \mathrm{~d} x\right)^{\frac{2}{3}}\left(\int_{\mathbb{R}^{N}}\left|u_{n}-u_{0}\right|^{6} \mathrm{~d} x\right)^{\frac{1}{6}} \\
& \leqslant C\left\|u_{n}-u_{0}\right\|_{L^{6}} \rightarrow 0,
\end{aligned}
$$

similarly, we have

$$
\left.\left|\int_{\mathbb{R}^{N}}\right| v_{n}|| \nabla v_{n}\right|^{2}\left(v_{n}-v_{0}\right) \mathrm{d} x \mid \rightarrow 0
$$

By Hölder inequality, we obtain

$$
\left|\int_{\mathbb{R}^{N}} u_{n}^{2}\right| \nabla u_{n}\left|\left(\nabla u_{n}-\nabla u_{0}\right) \mathrm{d} x\right| \leqslant\left(\int_{\mathbb{R}^{N}}\left|u_{n}\right|^{3} \mathrm{~d} x\right)^{\frac{2}{3}}\left(\int_{\mathbb{R}^{N}}\left|\nabla u_{n}\right|^{6} \mathrm{~d} x\right)^{\frac{1}{6}}\left(\int_{\mathbb{R}^{N}}\left|\nabla u_{n}-\nabla u_{0}\right|^{6} \mathrm{~d} x\right)^{\frac{1}{6}} \rightarrow 0 .
$$

Analogously, we get

$$
\left|\int_{\mathbb{R}^{N}} v_{n}^{2}\right| \nabla v_{n}\left|\left(\nabla v_{n}-\nabla v_{0}\right) \mathrm{d} x\right| \rightarrow 0
$$

By (3.10)-(3.11) we have

$$
\lim _{n \rightarrow \infty}\left\|\left(u_{n}, v_{n}\right)\right\|_{\lambda_{n}}^{2}=\lim _{n \rightarrow \infty}\left\langle\left(u_{n}, v_{n}\right),\left(u_{0}, v_{0}\right)\right\rangle_{\lambda_{n}}=\lim _{n \rightarrow \infty}\left\langle\left(u_{n}, v_{n}\right),\left(u_{0}, v_{0}\right)\right\rangle=\left\|\left(u_{0}, v_{0}\right)\right\|^{2} .
$$

On the other hand, weakly lower semi-continuity of norm yields that

$$
\left\|\left(u_{0}, v_{0}\right)\right\|^{2} \leqslant \lim _{n \rightarrow \infty} \inf \left\|\left(u_{n}, v_{n}\right)\right\|^{2} \leqslant \lim _{n \rightarrow \infty} \sup \left\|\left(u_{n}, v_{n}\right)\right\|^{2} \leqslant \lim _{n \rightarrow \infty}\left\|\left(u_{n}, v_{n}\right)\right\|_{\lambda_{n}}^{2} .
$$

This shows that $\left(u_{n}, v_{n}\right) \rightarrow\left(u_{0}, v_{0}\right)$ in $H^{2}\left(\mathbb{R}^{N}\right) \times H^{2}\left(\mathbb{R}^{N}\right)$. Finally, we can get $\left(u_{0}, v_{0}\right)$ is a nontrivial, and the proof is the similar to Theorem 1.1. This completes the proof.

\section{Acknowledgment}

This work is partially supported by Natural Science Foundation of China 11671403 and by the Fundamental Research Funds for the Central Universities of Central South University 2017zzts056.

\section{References}

[1] P. Álvarez-Caudevilla, E. Coloradoa, V. A. Galaktionov, Existence of solutions for a system of coupled nonlinear stationary bi-harmonic Schrödinger equations, Nonlinear Anal., 23 (2015), 78-93. 1

[2] T. Bartsch, Z. Q. Wang, Existence and multiplicity results for superlinear elliptic problems on $\mathrm{R}^{\mathrm{N}}, \mathrm{Comm}$. Partial Differential Equations, 20 (1995), 1725-1741. 1

[3] H. Brézis, E. Lieb, A relation between point convergence of functions and convergence of functionals, Proc. Amer. Math. Soc., 88 (1983), 486-490.

[4] P. Candito, L. Li, R. Livrea, Infinitely many solutions for a perturbed nonlinear Navier boundary value problem involving the p-biharmonic, Nonlinear Anal., 75 (2012), 6360-6369. 
[5] S. Chen, J. Liu, X. Wu, Existence and multiplicity of nontrivial solutions for a class of modified nonlinear fourth-order elliptic equations on $\mathbb{R}^{\mathrm{N}}$, Appl. Math. Comput., 248 (2014), 593-608. 1, 2

[6] Y. Chen, P. J. McKenna, Traveling waves in a nonlinear suspension beam: theoretical results and numerical observations, J. Differ. Equ., 136 (1997), 325-355. 1

[7] Q.-H. Choi, T. Jung, Multiplicity of solutions and source terms in a fourth order nonlinear elliptic equation, Acta Math. Sci., 19 (1999), 361-374. 1

[8] Z.-Y. Deng, Y.-S. Huang, Symmetric solutions for a class of singular biharmonic elliptic systems involving critical exponents, Appl. Math. Comput., 264 (2015), 323-334.

[9] M. Hajipour, A. Malek, High accurate NRK and MWENO scheme fornonlinear degenerate parabolic PDEs, Appl. Math. Model., 36 (2012), 4439-4451.

[10] M. Hajipour, A. Malek, High accurate modified WENO method for the solution of Black-Scholes equation, Comput. Appl. Math., 34 (2015), 125-140.

[11] Y.-S. Huang, X.-Q. Liu, Sign-changing solutions for p-biharmonic equations with Hardy potential in the half-space, J. Math. Anal. Appl., 444 (2016), 1417-1437. 1

[12] A. C. Lazer, P. J. McKenna, Large-amplitude periodic oscillations in suspension bridges: some new connections with nonlinear analysis, SIAM Rev., 32 (1990), 537-578. 1

[13] A. C. Lazer, P. J. McKenna, Global bifurcation and a theorem of Tarantello, J. Math. Anal. Appl., 181 (1994), 648-655. 1

[14] H. Liu, H. Chen, Least energy nodal solution for quasilinear biharmonic equations with critical exponent in $\mathbb{R}^{\mathrm{N}}$, Appl. Math. Lett., 48 (2015), 85-90. 1

[15] H. Liu, H. Chen, Ground-state solution for a class of biharmonic equations including critical exponent, Z. Angew. Math. Phys., 66 (2015), 3333-3343.

[16] D. Lü, Multiple solutions for a class of biharmonic elliptic systems with Sobolev critical exponent, Nonlinear Anal., 74 (2011), 6371-6382. 1

[17] P. J. McKenna, W. Walter, Traveling waves in a suspension bridge, SIAM J. Appl. Math., 50 (1990), 703-715. 1

[18] M. Millem, Minimax Theorems, Birkhäuser, Berlin, (1996). 1, 3

[19] M. T. O. Pimenta, S. H. M. Soares, Existence and concentration of solutions for a class of biharmonic equations, J. Math. Anal. Appl., 390 (2012), 274-289. 1

[20] L.-Y. Shao, H. Chen, Multiple solutions for Schrödinger-Poisson systems with sign-changing potential and critical nonlinearity, Electron. J. Differential Equations, 2016 (2016), 8 pages.

[21] L.-Y. Shao, H. Chen, Existence and concentration result for a quasilinear Schrödinger equation with critical growth, Z. Angew. Math. Phys., 2017 (2017), 16 pages.

[22] H. Shi, H. Chen, Existence and multiplicity of solutions for a class of generalized quasilinear Schrödinger equations, J. Math. Anal. Appl., 452 (2017), 578-594.

[23] J. Sun, J. Chu, T.-F. Wu, Existence and multiplicity of nontrivial solutions for some biharmonic equations with p-Laplacian, J. Differential Equations, 262 (2017), 945-977. 1

[24] A. Szulkin, T. Weth, Ground state solutions for some indefinite variational problems, J. Funct. Anal., 257 (2009), $3802-$ 3822. 1

[25] N. S. Trudinger, On Harnack type inequalities and their application to quasilinear elliptic equations, Comm. Pure Appl. Math., 20 (1967), 721-747. 3

[26] F. Wang, M. Avci, Y. An, Existence of solutions for fourth order elliptic equations of Kirchhoff type, J. Math. Anal. Appl., 409 (2014), 140-146. 1

[27] Y.-J. Wang, Y.-T. Shen, Multiple and sign-changing solutions for a class of semilinear biharmonic equation, J. Differential Equations, 246 (2009), 3109-3125.

[28] H. Xiong, Y.-T. Shen, Nonlinear biharmonic equations with critical potential, Acta Math. Sci., 21 (2005), $1285-1294$.

[29] J. Zhang, S. Li, Multiple nontrivial solutions for some fourth-order semilinear elliptic problems, Nonlinear Anal., 60 (2005), 221-230.

[30] W. Zhang, X. Tang, J. Zhang, Infinitely many solutions for fourth-order elliptic equations with general potentials, J. Math. Anal. Appl., 407 (2013), 359-368.

[31] J. Zhang, X. Tang, W. Zhang, Infinitely many solutions of quasilinear Schrödinger equation with sign-changing potential, J. Math. Anal. Appl., 420 (2014), 1762-1775.

[32] W. Zhang, X. Tang, J. Zhang, Infinitely many solutions for fourth-order elliptic equations with sign-changing potential, Taiwanese J. Math., 18 (2014), 645-659. 1

[33] W. Zhang, X. Tang, J. Zhang, Existence and concentration of solutions for sublinear fourth-order elliptic equations, Electron. J. Differential Equations, 2015 (2015), 9 pages. 1

[34] W. Zou, M. Schechter, Critical Point Theory and its Applications, Springer, New York, (2006). 2 